\title{
Impacto dos Valores Laborais e da Interferência Família - Trabalho no Estresse Ocupacional ${ }^{1}$
}

\author{
Tatiane Paschoal ${ }^{2}$ \\ Alvaro Tamayo \\ Universidade de Brasília
}

\begin{abstract}
RESUMO - Esta pesquisa teve como objetivo investigar a influência da interferência família-trabalho e dos valores do trabalho sobre o estresse ocupacional. Para tanto, utilizaram-se três instrumentos: Escala de Estresse no Trabalho, Escala de Interação Trabalho-Família e Escala de Valores Relativos ao Trabalho. Participaram da pesquisa 237 funcionários de uma instituição bancária. Os resultados indicaram que a interferência família-trabalho influencia o estresse ocupacional, sendo que quanto maior o escore de interferência, maior o estresse. Sugere-se que a interferência família-trabalho possa favorecer diretamente o aparecimento de estressores organizacionais e orientar cognições e afetos que influenciem a percepção de demandas do trabalho como estressores. Os valores do trabalho não apresentaram relação com o estresse ocupacional, o que pode ser decorrente do instrumento utilizado para avaliar o estresse ou da maior importância desses valores na escolha da profissão. Limitações e orientações para pesquisas futuras são apresentadas no texto.
\end{abstract}

Palavras-chave: estresse ocupacional; valores do trabalho; interferência família-trabalho.

\section{Impact of Work Values and Family - Work Interference on Occupational Stress}

\begin{abstract}
The objective of this research was to investigate the influence of family-work interference and work values on occupational stress. Three instruments were used to collect data: Work Stress Scale, the Work-Family Interaction Scale and the Work Values Scale. The instruments were administered to 237 workers of a banking institution. The results showed that the family-work interference did influence on occupational stress in the sense that higher scores on interference were correlated with higher stress. The authors suggest that family-work interference can directly contribute to the emergence of organizational stressors and influence cognition and affects that can have an impact on the person's perception of job demands as stressors. Work values had no relation with occupational stress. This could be explained by the instrument used to evaluate occupational stress or the importance given to work values in the process of professional choice. Limitations and orientations to future research are pointed.
\end{abstract}

Key words: occupational stress; work values; family-work interference.

Muitos esforços têm sido alocados para investigar variáveis que influenciam o estresse ocupacional, fenômeno constantemente associado à saúde do trabalhador e ao desempenho organizacional. Basicamente, o estresse ocupacional pode ser definido com ênfase nos fatores do trabalho que excedem a capacidade de enfrentamento do indivíduo (estressores organizacionais) ou nas respostas fisiológicas, psicológicas e comportamentais dos indivíduos aos estressores (Jex, 1998; Jones \& Kinman, 2001).

Essas duas concepções, porém, recebem críticas diversas (Beehr, 1998; Jex, 1998) e têm sido preteridas em função de definições mais abrangentes. Para Beehr (1998), por exemplo, o estresse ocupacional deveria ser concebido como uma área de estudo e prática que se preocupa com diversas variáveis interligadas, tais como estímulos do ambiente de trabalho e respostas não saudáveis de pessoas expostas a eles. A tendência nos últimos anos, portanto, tem sido de considerar

1 Apoio do Conselho Nacional de Desenvolvimento Científico e Tecnológico - CNPq.

2 Endereço: Universidade de Brasília, Campus Darcy Ribeiro, Instituto de Psicologia, Depto. de Psicologia Social e do Trabalho, Brasília, DF, Brasil 70910-900.E-mail: tatianepaschoal@yahoo.com.br o estresse ocupacional como um processo concebido por estressores e respostas e como um conceito relacional, o qual enfoca a relação entre o ambiente de trabalho e o sujeito. Isto pode ser observado, por exemplo, na teoria de Lazarus (1995), na teoria cibernética do estresse (Edwards, 1992) e na teoria de ajustamento pessoa-ambiente de Edwards e Cooper (1990).

Lazarus (1995) propõe que o estresse ocupacional ocorre quando o indivíduo avalia as demandas do trabalho como excessivas para os recursos de enfrentamento que possui. $\mathrm{O}$ estresse não seria uma propriedade da pessoa ou do ambiente, mas poderia se desenvolver a partir da conjunção de um tipo particular de ambiente com um tipo determinado de pessoa. Pressão de tempo, sobrecarga de trabalho, falta de autonomia e conflitos com superiores certamente são estressores para um grande número de trabalhadores, mas não necessariamente para todos. Certos tipos de pessoas, como aquelas que têm tendência à depressão, por exemplo, teriam tendência a reagir mais vezes ou mais intensamente aos estressores organizacionais, o que resultaria em problemas como absenteísmo, baixo desempenho e disfunções emocionais.

A teoria cibernética (Edwards, 1992) tem como princípio básico o negative feedback loop, em que um indivíduo recebe 
informações do ambiente e as compara com um critério de referência relevante. Se a comparação indica uma discrepância entre o ambiente percebido e o critério de referência, o indivíduo tenta alterar o ambiente para diminuir ou eliminar a discrepância. Este processo pode ser iniciado por um distúrbio no ambiente ou por uma mudança no critério de referência do indivíduo. O estresse ocupacional, na teoria cibernética, pode ser entendido como uma discrepância entre uma situação percebida pelo empregado e uma situação desejada, desde que a presença da discrepância seja considerada importante pelo empregado.

Com relação à teoria de ajustamento pessoa-ambiente (person-environment fit), Edwards e Cooper (1990) operacionalizam o estresse ocupacional calculando a discrepância entre o que o indivíduo percebe e o que deseja de vários aspectos do contexto do trabalho. A idéia por trás desta teoria é de que o comportamento seria uma função da interação entre a pessoa e o ambiente, sendo que um aspecto desta relação consistiria no grau em que o indivíduo ajusta-se à situação. Tanto a teoria de ajustamento pessoa-ambiente (Edwards \& Cooper, 1990) quanto a teoria cibernética (Edwards, 1992) consideram o estresse ocupacional como uma relação entre o indivíduo e seu ambiente de trabalho, caracterizada por uma discrepância entre o que o indivíduo deseja e o que percebe como sendo real. A teoria cibernética, porém, ressalta o critério de referência relevante do indivíduo, isto é, a presença da discrepância deve ser considerada importante pelo empregado.

Entre a variedade de teorias que conceituam o estresse ocupacional como um processo e enfocam seu caráter relacional, tem havido consenso em considerar as percepções individuais como mediadoras de suma importância do impacto do ambiente de trabalho sobre as respostas do indivíduo (Cooper, Dewe \& O’ Driscoll, 2001; Edwards, 1992; Edwards \& Cooper, 1990; Jex, 1998; Kahn e Byosiere, 1992; Lazarus, 1995). Paschoal e Tamayo (2004) conceituam o estresse ocupacional como um processo em que o indivíduo percebe demandas do trabalho como estressores, os quais, ao exceder sua habilidade de enfrentamento, provocam no sujeito reações negativas. Esta definição foi considerada para abordar o estresse ocupacional no presente estudo.

\section{Variáveis que influenciam o estresse ocupacional}

Sendo a percepção das demandas como estressores um elemento central para a compreensão do estresse, faz-se relevante o estudo de variáveis que podem ter impacto neste processo. Nas duas últimas décadas, inúmeras pesquisas têm investigado variáveis de natureza situacional e pessoal que podem influenciar o estresse ocupacional.

No âmbito das variáveis situacionais, o suporte social encontrado dentro e fora do trabalho tem sido constantemente associado ao estresse ocupacional. Estudos apontam que a presença de redes sociais que oferecem suporte ao trabalho melhora a avaliação do empregado sobre sua saúde e correlaciona-se negativamente com o estresse (Browner, 1987; Iwata \& Suzuki, 1997). Segundo Tamayo, Lima e Silva (2002), o impacto do suporte social sobre o estresse ocupacional pode ser benéfico ou prejudicial, dependendo da qualidade desta dimensão na vida cotidiana da organização. Quando o suporte social está bem desenvolvido na organização, ele tem um efeito protetor que se manifesta em baixos níveis de estresse, ou seja, quanto maior o nível de suporte social no ambiente organizacional, menor o nível de estresse no trabalho. Por outro lado, quando o suporte social é inexistente ou deficitário na organização, este fator transforma-se num estressor.

Variáveis situacionais que não se restringem a aspectos organizacionais, como a atividade física regular, também parecem ter uma influência sobre o estresse ocupacional (Tamayo, 2001). Trabalhadores que não praticam exercícios físicos regularmente tendem a apresentar um nível de estresse superior. O exercício físico regular desenvolve o condicionamento cardíaco que provoca, na corrente sanguínea, uma redução de substâncias associadas ao estresse. Além da dimensão fisiológica, destaca-se a dimensão psicossocial de várias modalidades de atividade física, sendo que a interação social e a comunicação interpessoal podem servir de estratégias para lidar com situações estressantes (Tamayo, 2001).

A interação entre trabalho e família também é alvo do interesse de pesquisadores da área. Para alguns autores, a existência de conflito entre papéis desempenhados nessas duas dimensões pode ser tratada como um estressor organizacional (Cooper, Sloan \& Williams, 1988). Considerando-se os estressores organizacionais como demandas do ambiente de trabalho que exigem respostas adaptativas por parte do empregado e que excedem sua habilidade de enfrentamento, aspectos da relação entre trabalho e família podem ser tratados como variáveis situacionais que influenciam o estresse ocupacional. Isto porque não se restringem a demandas do ambiente de trabalho, mas podem influenciar a percepção dos estressores. Na maioria das vezes, investigam-se quais impactos o estresse ocupacional pode ter na família e como se dá esse processo (Perry-Jenkins, Repetti \& Crouter, 2000). A direção contrária, porém, também pode ser estabelecida, ou seja, de que modo acontecimentos na família podem ter impacto na vida profissional do indivíduo (Frone, Yardley \& Markel, 1997; MacEwen \& Barling, 1994), o que tem sido menos explorado.

Como apontado anteriormente, características individuais dos trabalhadores também têm permeado os estudos na área. De acordo com Kahn e Byosiere (1992), a consideração de características e diferenças individuais para o entendimento do comportamento humano é um fenômeno antigo. $\mathrm{Na}$ área de estresse ocupacional, apesar de a ênfase residir no estilo de enfrentamento (estilo de coping) do empregado frente aos eventos estressores, variáveis como o locus de controle, o padrão de comportamento tipo A e a auto-estima também têm chamado a atenção dos pesquisadores.

Quanto ao locus de controle, a suposição geral é de que pessoas com locus de controle interno respondem ao estresse diferentemente daquelas com locus de controle externo (Marino \& White, 1985). Aqueles orientados pela internalidade tendem a agir contra as fontes de estresse e providenciar formas de minimizar seus efeitos, enquanto aqueles mais externos comportam-se de forma mais passiva, ficando mais suscetíveis ao impacto dos estressores.

Em relação ao padrão de comportamento tipo A, dados demonstram que estes indivíduos (competitivos, impacientes e agressivos) tendem a experimentar mais conseqüências negativas diante de demandas laborais do que os indivíduos 
tipo B, trabalhando em condições semelhantes (Caplan \& Jones, 1975; Jamal, 1990). Algumas pesquisas, porém, não têm encontrado efeito do comportamento tipo A sobre o estresse ocupacional (Kirmeyer \& Diamond, 1985; Schmied \& Lawler, 1986). Segundo Kahn e Byosiere (1992), a divergência entre esses estudos deve-se à utilização de instrumentos pouco confiáveis de comportamento tipo A e medidas limitadas de estressores organizacionais e respostas negativas dos trabalhadores. Para os autores, parece que o comportamento tipo A correlaciona-se apenas com alguns tipos de estressores, como aqueles referentes ao relacionamento interpessoal no trabalho, e determinados tipos de respostas, como ansiedade. Dessa forma, a falta de correlação entre padrão de comportamento e estresse ocupacional pode ocorrer quando a medida de estresse não engloba estressores de relacionamento e consequiências como ansiedade.

Outra variável pessoal considerada nos estudos sobre estresse ocupacional consiste na auto-estima. Segundo Kunda (1999), a auto-estima pode ser definida como um construto estável que influencia as estratégias cognitivas utilizadas pelo indivíduo no processamento de informações relevantes sobre si mesmo e suas reações a essas informações. Segundo Tamayo (2001), a auto-estima desempenha uma função importante na proteção do self e, quando elevada, constitui-se num filtro protetor que seleciona as informações referentes ao indivíduo. Nos estudos sobre estresse, a auto-estima tem sido vista como um recurso para o processo de enfrentamento, capaz de influenciar a avaliação dos eventos e os comportamentos emitidos para lidar com os estressores. Segundo Jex e Elacqua (1999), indivíduos com baixa auto-estima tendem a utilizar formas mais passivas de enfrentamento, o que faz com que fiquem mais vulneráveis aos efeitos dos estressores. No geral, parece haver uma correlação positiva entre baixa auto-estima e altos níveis de estresse ocupacional.

Outras variáveis pessoais também foram relacionadas ao estresse ocupacional, como por exemplo, as prioridades axiológicas do indivíduo. Inúmeros estudos têm demonstrado o papel preditivo dos valores sobre o comportamento organizacional. Considerando as prioridades axiológicas como princípios e metas, organizados hierarquicamente, que orientam a forma de pensar e de agir do indivíduo, Tamayo (2001) investigou a relação entre as prioridades axiológicas e os níveis de estresse ocupacional. Dez tipos motivacionais foram considerados na medida de valores. O autor verificou que o nível de estresse foi maior para indivíduos com maior escore nos tipos motivacionais realização e hedonismo. Estes dois tipos motivacionais expressam interesses individuais e são adjacentes, o que significa que têm metas compatíveis. Segundo Tamayo (2001), "a meta dos valores de realização é a procura de sucesso pessoal obtido por meio de uma demonstração de competência pessoal reconhecida socialmente; a meta dos valores de hedonismo é a procura de prazer e de gratificação sensual" (p. 139). De acordo com a pesquisa de Tamayo (2001), os sujeitos que enfatizam a realização pessoal e o hedonismo, são mais suscetíveis ao estresse no trabalho.

A partir da revisão das variáveis que predizem estresse ocupacional, lacunas podem ser apontadas. Por exemplo, quanto ao nível situacional, sabe-se que relações entre trabalho e família podem ajudar na compreensão de fenôme- nos organizacionais, mas na área de estresse ocupacional, enfocam-se os impactos ou as interferências do ambiente de trabalho sobre a família. No âmbito individual, a revisão de literatura no Brasil apontou uma única pesquisa, já referida anteriormente, relacionando valores e estresse, a qual demonstrou a importância dos valores pessoais na percepção do estresse ocupacional (Tamayo, 2001). Não foram encontrados, porém, estudos relacionando o estresse ocupacional com os valores relativos ao trabalho, mais específicos desta esfera da vida do indivíduo.

Esta pesquisa, portanto, teve como objetivos: a) investigar o impacto da interferência família-trabalho sobre o estresse ocupacional e b) investigar o impacto dos valores do trabalho sobre o estresse ocupacional.

\section{Interferência família-trabalho}

Diversos são os modelos apresentados para explicar as formas de relação entre trabalho e família. Segundo Edwards e Rothbard (2000), os mecanismos de interação entre essas duas dimensões podem ser divididos em seis categorias: contaminação (trabalho e família são similares, havendo um impacto de uma dimensão sobre a outra), compensação (a insatisfação num domínio leva a pessoa a aumentar seu envolvimento ou procurar recompensas no outro), segmentação (separação do trabalho e da família, de modo que um domínio não influencia o outro), escoamento de recursos (recursos como tempo, atenção e energia são limitados e aqueles despendidos num domínio ficam indisponíveis para outro) e conflito (demandas do trabalho e da família são mutuamente incompatíveis, de modo que cumprir as demandas em um domínio dificulta o cumprimento em outro). Com exceção dos modelos de conflito, que contam com dados mais consistentes, muitos dos modelos ainda costumam ser tratados em nível teórico e carecem de dados empíricos. Apesar disso, parece haver consenso entre os autores sobre duas formas básicas de relação entre as duas esferas. A primeira diz respeito ao impacto do trabalho sobre a família e a segunda refere-se ao impacto da família sobre o trabalho (Frone, Russell \& Cooper, 1992; Kelloway, Gottlieb \& Barham, 1999). Os determinantes da natureza dos impactos causados pela combinação do trabalho doméstico e profissional incluem as demandas em cada esfera, o nível de apoio com o qual o indivíduo pode contar para atender às responsabilidades nas duas esferas e os mecanismos de apoio existentes na comunidade.

De modo geral, as interações negativas entre trabalho e família têm recebido especial atenção dos pesquisadores. Um conceito bastante investigado, por exemplo, é o de conflito entre papéis profissionais e familiares (Frone \& cols., 1992; Kelloway \& cols., 1999). De acordo com Polasky e Holahan (1998), a literatura mostra a importância, para a saúde e bemestar do indivíduo, de se desempenhar diversos papéis dentro e fora de casa, pois isso aumenta a auto-estima e oferece maiores oportunidades de apoio social. No entanto, estudos têm revelado que tentativas de equilibrar demandas advindas do trabalho e da família podem provocar conseqüências negativas no âmbito familiar e profissional (CARNET, 1993; Thompson \& Walker, 1989). Assim, é possível compreender a preocupação com a investigação de como se dão os impactos 
negativos de uma esfera sobre outra e os efeitos sobre o indivíduo, sua família e seu ambiente de trabalho.

Segundo Perry-Jenkins e cols. (2000), estudos que se preocuparam em investigar os impactos da família sobre trabalho sugerem que relações familiares conturbadas e estresse relacionado à vida conjugal tendem a se relacionar positivamente com absenteísmo e negativamente com desempenho no trabalho. Frone e cols. (1997) e MacEwen e Barling (1994) investigaram a relação entre conflitos de família com trabalho e comportamentos organizacionais. Os resultados mostraram que quando acontecimentos e demandas familiares começam a interferir nas demandas do trabalho e entrar em conflito com elas, o desempenho no trabalho tende a diminuir (Frone \& cols., 1997) e o desgaste no trabalho a aumentar (MacEwen \& Barling, 1994).

Tamayo e cols. (2002) investigaram o poder preditivo da interferência do trabalho na família e da família no trabalho sobre o autoconceito profissional. Este construto refere-se à percepção do indivíduo sobre si mesmo em relação ao trabalho que executa. As dimensões avaliadas do autoconceito profissional foram: segurança profissional (percepção do indivíduo sobre sua segurança diante de situações diferentes e novas no trabalho); realização profissional (percepção do indivíduo sobre suas aspirações e ideais realizados por meio do trabalho executado); saúde no trabalho (percepção do indivíduo sobre como o trabalho ou os fatos que o envolvem podem afetar sua saúde física e mental) e competência no trabalho (percepção do indivíduo sobre sua competência no trabalho e sua contribuição para a organização em que trabalha). A saúde no trabalho foi explicada pela interferência trabalho-família. A interferência família-trabalho explicou os fatores segurança profissional e competência no trabalho. Em todos os casos, observou-se uma relação negativa entre as variáveis independentes e as dependentes, o que significa que quanto mais os acontecimentos do trabalho interferem na família, menor a percepção de saúde no trabalho e quanto mais os acontecimentos na família interferem na realização do trabalho, menor a percepção de segurança e competência profissional.

Considerando os dados que sugerem que interferências da família no trabalho têm impacto no contexto organizacional, foram selecionadas duas hipóteses para esta pesquisa:

Hipótese 1: é de que a interferência da família no trabalho deverá se relacionar com o estresse ocupacional, sendo que quanto maior o escore de interferência, maior o estresse. É conveniente destacar que não foram estabelecidas relações entre acontecimentos isolados na família (como por exemplo, o estresse na família, a sobrecarga na família e a satisfação na família) e o estresse ocupacional. Enfocou-se um tipo específico de interação trabalho-família: a interferência da família no trabalho, um tipo de interação em que eventos gerados na família começam a atrapalhar a realização do trabalho. $\mathrm{Na}$ pesquisa realizada por Frone e cols. (1992), observou-se que experiências familiares isoladas (como estressores familiares e comprometimento com deveres domésticos, por exemplo) não estavam diretamente relacionadas com experiências no trabalho. Estas eram modificadas quando os acontecimentos da família entravam em conflito com demandas do trabalho, de forma que experiências negativas no trabalho eram mais comuns quando havia conflito da família com o trabalho.
Muitos teóricos, de variados campos de estudo, têm defendido a relevância dos valores para a compreensão e previsão de atitudes e comportamentos. Partindo do pressuposto de que o trabalho é um dos aspectos básicos na vida do ser humano, alguns estudos procuram enfatizar a relação entre comportamentos e valores relativos ao trabalho.

Foi adotado na presente pesquisa o modelo de valores do trabalho de Porto e Tamayo (2003), desenvolvido a partir do modelo de Ros, Schwartz e Surkiss (1999), o qual se baseia na Teoria de Valores de Schwartz. Para Ros e cols. (1999), valores do trabalho podem ser definidos como crenças sobre metas e comportamentos desejáveis, hierarquicamente organizadas, que guiam as avaliações sobre os resultados e o contexto do trabalho, bem como a seleção de alternativas de trabalho. Segundo Porto e Tamayo (2003), assim como no caso dos valores pessoais, é possível estabelecer uma estrutura dos valores do trabalho, a qual recebeu suporte de pesquisas realizadas no Brasil. Essa estrutura constitui-se de quatro tipos de valores, os quais foram denominados de realização no trabalho, relações sociais, prestígio e estabilidade. O tipo motivacional realização no trabalho refere-se à busca de realização pessoal e profissional, bem como de independência de pensamento e ação no trabalho por meio da autonomia intelectual e da criatividade. Como exemplo de valores pertencentes a esta categoria pode-se citar: trabalho intelectualmente estimulante, ter prazer no que faço, realizar um trabalho significativo para mim e identificar-me com o trabalho. O tipo motivacional relações sociais refere-se à busca de relações sociais positivas no trabalho e de contribuições positivas para a sociedade por meio do trabalho. Exemplo de valores: auxiliar os colegas de trabalho, amizade com colegas de trabalho, colaborar para o desenvolvimento da sociedade e combater injustiças sociais. O tipo motivacional prestígio refere-se à busca de autoridade, sucesso profissional e poder de influência no trabalho. Como exemplo de valores, pode-se apontar: obter posição de destaque, ter fama, supervisionar outras pessoas e competir com colegas de trabalho para alcançar as minhas metas profissionais. Por fim, o tipo motivacional estabilidade expressa a busca de segurança e ordem na vida por meio do trabalho, possibilitando suprir materialmente as necessidades pessoais; esse tipo motivacional refere-se à busca de recompensas extrínsecas ao trabalho. Exemplo de valores: estabilidade financeira, ter melhores condições de vida, ser independente financeiramente e poder me sustentar.

Esses quatro tipos de valores do trabalho têm, de acordo com a definição, a característica de guiar as avaliações sobre os resultados e o contexto de trabalho. A compreensão do estresse ocupacional, por sua vez, tem se apoiado na percepção do indivíduo, que avalia suas demandas de trabalho e os acontecimentos na organização. É possível, portanto, que os valores do trabalho, como orientadores de avaliações individuais, estejam relacionados com o estresse ocupacional. Indivíduos com que tipo de hierarquia de valores do trabalho estariam mais suscetíveis a perceber acontecimentos do trabalho como estressores? De acordo com a revisão de literatura, esta questão não foi ainda explorada, tornando-se difícil estabelecer hipóteses sobre o impacto de cada tipo de valor do trabalho sobre o estresse. Por outro lado, a idéia de que os valores laborais podem ter impacto sobre a percepção 
de estressores organizacionais tem relativo suporte teórico. Assim, a segunda hipótese desta pesquisa é:

Hipótese 2: os valores do trabalho terão impacto sobre o estresse ocupacional.

\section{Método}

\section{Participantes}

Participaram da pesquisa 237 funcionários de uma instituição bancária, entre homens $(64,6 \%)$ e mulheres $(35,4 \%)$, com média de idade de 39,15 anos $(D P=7,41)$ e grau de instrução variando de segundo grau completo $(8,0 \%)$ a terceiro grau completo ou pós-graduação $(70,1 \%)$. Dentre os participantes, $71,3 \%$ eram casados e $16,5 \%$, solteiros, sendo que $74,3 \%$ tinham filhos. A média de tempo de serviço na organização foi de 17,1 anos $(D P=7,11)$. Quanto à função desempenhada, 35, 4\% eram analistas, 30,0\%, gerentes e $19,4 \%$ eram assessores, sendo que o restante dividiu-se entre assistentes e outros cargos administrativos.

\section{Instrumentos}

Utilizou-se um questionário composto a partir de três escalas: Escala de Estresse no Trabalho, Escala de Interação Trabalho-Família e Escala de Valores Relativos ao Trabalho. A Escala de Estresse no Trabalho, validada por Paschoal e Tamayo (2004), é composta por 23 itens, os quais formam um único fator. Consiste numa medida geral de estresse, cujos itens abordam estressores variados e reações emocionais constantemente associadas aos mesmos. Os itens deveriam ser avaliados de acordo com uma escala de concordância de cinco pontos $(1-$ discordo totalmente a 5 - concordo totalmente), sendo que quanto maior a pontuação, maior o estresse. $\mathrm{O}$ índice de confiabilidade da escala - alpha de Cronbach $(\alpha)$ - foi de 0,93 .

Para avaliar a interferência da família no trabalho, utilizou-se a Escala de Interação Trabalho-Família, validada por Paschoal, Tamayo e Barham (2002). Este instrumento é composto por dois fatores: impacto da família sobre o trabalho e impacto do trabalho sobre a família, ambos enfocando as interferências de um sobre outro. Cada fator é formado por sete itens que deveriam ser respondidos a partir de uma escala de frequiência que variava de 1 (nunca) a 5 (quase sempre). Para este estudo, foram avaliadas as respostas referentes ao fator de interferência da família no trabalho, o qual apresentou um $\alpha$ de 0,76 .

Os quatro tipos de valores do trabalho foram avaliados por meio da Escala de Valores Relativos ao Trabalho, validada por Porto e Tamayo (2003) e composta pelos seguintes fatores: realização no trabalho $(\alpha$ de 0,89$)$; relações sociais ( $\alpha$ de 0,90); prestígio ( $\alpha$ de 0,83); estabilidade ( $\alpha$ de 0,81). Ao todo são 45 itens que deveriam ser respondidos de acordo com uma escala de importância que variava de 1 (nada importante) a 5 (extremamente importante).

\section{Procedimento}

A coleta de dados foi realizada no centro de treinamento da organização participante da pesquisa. Os questionários foram aplicados coletivamente em funcionários que participavam de algum treinamento oferecido pela empresa. Um pesquisador entrava na sala de treinamento, geralmente após o intervalo, e distribuía os questionários para os participantes. Os questionários eram respondidos e, em seguida, devolvidos ao pesquisador. Critérios éticos foram considerados: os participantes foram informados da natureza da pesquisa, podiam optar por participar ou não e tiveram seu anonimato garantido.

\section{Análise dos dados}

Como não havia informações teóricas e/ou empíricas para prever quais das variáveis independentes seriam preditoras da variável dependente, foi realizada uma regressão múltipla Stepwise. Na regressão Stepwise, a ordem de entrada das variáveis está baseada exclusivamente em critérios estatísticos, sem nenhuma intervenção de critérios teóricos ou de preferências do pesquisador. A variável dependente foi o estresse ocupacional e as variáveis independentes foram os quatro fatores dos valores do trabalho e a interferência família-trabalho. Realizou-se também Teste-t.

\section{Resultados}

Em relação ao estresse ocupacional, a média da amostra foi de 2,59 ( $D P=0,77$; mínimo de 1,05 e máximo de 4,64). A média para a interferência família-trabalho foi de 1,95 $(D P=0,48)$. Para os valores do trabalho foram encontrados os seguintes resultados: estabilidade (média de 4,28; $D P=0,50$ ); realização no trabalho (média de 4,21; $D P=0,45)$; relações sociais (média de 3,$87 ; D P=0,63$ ); prestígio (média de 2,$72 ; D P=0,79$ ).

Os resultados da regressão múltipla mostraram que somente a variável interferência família-trabalho explicou a variável estresse ocupacional. A correlação entre essas duas variáveis foi positiva e o $R^{2}$ ajustado foi de 0,084 $(p<0,001)$. Os valores do trabalho (realização no trabalho, relações sociais, prestígio e estabilidade) não entraram no modelo de preditores. A Tabela 1 apresenta os coeficientes de regressão não padronizados $(\mathrm{B})$, os coeficientes de regressão padronizados $(\beta)$, o $R^{2}$ e o $R^{2}$ ajustado em relação à interferência família-trabalho.

Tabela 1. Regressão da variável interferência família-trabalho sobre o estresse ocupacional

\begin{tabular}{ccccc}
\hline Variável preditora & $\mathbf{B}$ & $\beta$ & $\mathbf{R}^{2}$ & $\begin{array}{c}R^{2} \\
\text { ajustado }\end{array}$ \\
\hline Interferência família-trabalho & 0,452 & 0,297 & 0,088 & 0,084 \\
\hline
\end{tabular}

Como não foi encontrada relação entre os valores do trabalho e o estresse ocupacional a partir da regressão múltipla, foi realizado um Test-t para comparar o grupo de respondentes com escore alto em estresse ocupacional e o grupo de respondentes com escore baixo. O primeiro grupo ficou composto por sujeitos com média de estresse ocupacional que variava de 3 a $5(N=51)$. O segundo grupo foi formado por sujeitos com média variando de 1 a $2(N=$ 61). O percentil superior foi $74 \mathrm{e} \mathrm{o} \mathrm{inferior,} 25$. O Teste-t foi realizado para cada um dos fatores de valores do trabalho, 
mas não se constatou diferença significativa entre os grupos com escores alto e baixo de estresse ocupacional.

\section{Discussão}

\section{Hipótese 1}

O primeiro objetivo desta pesquisa consistiu em investigar o impacto da interferência família-trabalho sobre o estresse ocupacional. A hipótese formulada para esta questão foi de que a interferência da família no trabalho estaria positivamente relacionada com o estresse ocupacional, ou seja, quanto maior o escore de interferência famíliatrabalho, maior o de estresse. Os resultados encontrados confirmaram essa hipótese e vão ao encontro de pesquisas anteriores sobre o tema.

Por um lado, a interferência família-trabalho pode ter uma participação direta no processo do estresse ocupacional. Os itens que avaliaram a interferência família-trabalho enfocaram eventos como: mudar os planos no trabalho para atender a compromissos familiares, sair mais cedo do trabalho para resolver problemas em casa e trabalhar de mau humor pensando na família. Interferências deste tipo podem comprometer o planejamento e a execução de tarefas profissionais, deixando estas suscetíveis a acontecimentos que independem da vontade do indivíduo. Este, por sua vez, tem demandas a cumprir, mas depara-se com acontecimentos familiares que interrompem seu trabalho e limitam sua atuação. Esta situação pode favorecer desentendimentos entre o funcionário e sua chefia, entre o funcionário e colegas que têm tarefas interligadas e comprometer a qualidade e a quantidade de trabalho realizado.

Todos esses eventos constituem potenciais estressores ligados a problemas de relacionamento interpessoal no trabalho e a sobrecargas qualitativa e quantitativa. Além disso, as interferências família-trabalho podem fazer com que o indivíduo perceba-se com pouco controle sobre suas responsabilidades profissionais, o que pode se constituir em outro estressor organizacional. Desta forma, a interferência família-trabalho estaria favorecendo, diretamente, o aparecimento de estressores organizacionais.

Por outro lado, a interferência família-trabalho pode atuar indiretamente no processo de estresse, favorecendo o aparecimento de afetos negativos, como irritação e ansiedade, que podem predispor o trabalhador a perceber os eventos organizacionais como estressores. Pesquisas têm mostrado que o estado de humor do indivíduo pode interferir nos julgamentos das situações com que se depara (Kunda, 1999). Assim, um desentendimento com um superior, uma dificuldade em realizar uma tarefa mais complexa e a existência de tarefas ambíguas ou conflituosas, por exemplo, podem passar a ser percebidos como estressores.

Nesta pesquisa, a porcentagem de variância do estresse ocupacional explicada pela interferência da família sobre o trabalho foi modesta $(8,4 \%)$. Este, no entanto, consiste em um resultado coerente com a conceituação da variável dependente. Em primeiro lugar, o estresse ocupacional configura-se como um fenômeno complexo, um processo que abrange diferentes variáveis interligadas, pessoais e organizacionais. Um modelo que explicasse melhor o estresse ocupacional deveria incluir uma quantidade de variáveis preditoras maior do que a considerada neste estudo. Em segundo lugar, o estresse ocupacional é basicamente determinado por condições do trabalho, por demandas que podem se constituir como estressores organizacionais. Juntas, as variáveis do ambiente de trabalho devem exercer um impacto importante sobre o estresse ocupacional. Neste estudo, abordou-se uma variável situacional não restrita ao ambiente de trabalho, mas que abrangia acontecimentos do âmbito familiar da vida dos indivíduos. Considerando-se a importância das variáveis organizacionais para o fenômeno em questão, o resultado encontrado para a interferência família-trabalho pode ser considerado relevante, pois se trata de variável extra-trabalho que tem um impacto em experiências relativas ao trabalho. A explicação de $8,4 \%$ de variância indica que acontecimentos familiares, quando começam a interferir no trabalho, podem ser favorecedores do aparecimento de estressores organizacionais e/ou potencializar percepções e afetos que orientam a avaliação de eventos laborais como estressores.

\section{Hipótese 2}

O segundo objetivo desta pesquisa consistiu em investigar o impacto dos valores do trabalho sobre o estresse ocupacional. A hipótese formulada para esta questão foi a de que haveria relação entre valores do trabalho e estresse ocupacional. Os resultados encontrados não sustentaram essa hipótese.

A suposição de que os valores do trabalho teriam influência sobre o estresse ocupacional partiu da definição daquele construto, segundo a qual, os valores do trabalho são crenças sobre metas e comportamentos desejáveis que guiam as avaliações do indivíduo sobre o contexto do trabalho. Dado que o estresse ocupacional depende da avaliação e do julgamento do indivíduo em relação aos eventos organizacionais como estressores, esperava-se que os valores do trabalho pudessem ter alguma relação com a percepção do estresse ocupacional.

Em sua revisão sobre a relação entre comportamento tipo A e estresse ocupacional, Kahn e Byosiere (1992) salientam as divergências entre os estudos que abordam esses dois construtos e apontam, como uma das justificativas para essa inconsistência, a utilização de medidas diferentes e limitadas de estressores organizacionais. Para os autores, a influência do comportamento tipo A sobre o estresse ocupacional ocorre apenas com alguns tipos de estressores. Há possibilidade de que, conforme sugerido por Kahn e Byosiere em relação ao comportamento tipo A, os valores do trabalho também se correlacionem com estressores organizacionais específicos.

A escala utilizada para avaliar o estresse ocupacional era global e formada por um único fator, cujos itens continham estressores variados e reações emocionais frequientemente associadas aos mesmos. No caso da consideração de estressores específicos, uma alta importância atribuída ao tipo motivacional relações sociais, por exemplo, poderia influenciar o estresse ocupacional associado a estressores de ordem interpessoal, como problemas no relacionamento com chefia e colegas do mesmo nível hierárquico. Do mesmo modo, a importância atribuída ao tipo motivacional realização no trabalho poderia influenciar o estresse ocupacional quando 
considerados estressores relativos ao desenvolvimento na carreira e autonomia no trabalho.

Algumas pesquisas sobre valores do trabalho e comportamentos organizacionais têm encontrado correlações baixas entre as duas variáveis. Konrad (2002), por exemplo, investigou a relação dos valores do trabalho com a participação de homens e mulheres em emprego remunerado e com a carga horária de trabalho semanal. Quanto à prática ou não de trabalho remunerado, foram constatadas associações fracas com os valores laborais. Estes tiveram, no caso das mulheres, impacto sobre o número de horas trabalhadas por semana, ainda que se tenham caracterizado como preditores pobres quando variáveis demográficas e responsabilidades familiares foram controladas. Arciniega e González (2002) avaliaram a influência dos valores do trabalho sobre o desenvolvimento do comprometimento organizacional. Os dados revelaram que cada dimensão desta variável era predita por diferentes dimensões de valores. Os autores salientam, porém, que a satisfação no trabalho e fatores organizacionais, como o conhecimento das metas da organização, apresentaram valor preditivo mais forte.

Há possibilidade de que os valores do trabalho tenham um papel preditivo relevante no processo de escolha profissional. O indivíduo avalia qual profissão ou emprego representa uma oportunidade para alcançar as metas que considera relevantes, ou seja, quais metas podem ser alcançadas por meio de determinado trabalho. Por exemplo, um indivíduo que atribua maior importância ao tipo motivacional estabilidade irá procurar profissões ou empregos que ofereçam um salário que satisfaça suas necessidades materiais e ofereçam estabilidade. Esses aspectos da profissão ou do emprego serão mais valorizados do que aspectos como autonomia, possibilidade de exercer a criatividade, exercer influência sobre os outros e colaborar com colegas de trabalho e com a sociedade em geral, os quais representam valores dos outros três tipos motivacionais.

Pode-se supor que a partir do momento em que o indivíduo escolhe o trabalho que vai desenvolver, entre as opções que tem disponíveis, e começa a fazê-lo, as metas são alcançadas. É possível que, para os respondentes da pesquisa, as metas referentes ao trabalho já tenham sido alcançadas quando eles entraram na organização e começaram a desenvolver suas atividades, sendo que o estresse ocupacional não seria influenciado pelos valores do trabalho. Particularmente, considerando a média de tempo de serviço da amostra que foi equivalente a 17,1 anos. Esta suposição não quer dizer que os indivíduos acomodem-se depois de seguir uma profissão ou conseguir um emprego que vai ao encontro de seus valores do trabalho; também não quer dizer que depois de um certo tempo de serviço, os indivíduos não desenvolvam novos interesses ou continuem avaliando situações de trabalho de acordo com seus valores. A suposição aqui apresentada sugere que se o indivíduo entrou num emprego que satisfaz suas metas, ou seja, se ele alcançou suas metas quando começou a trabalhar em determinado emprego e continua desenvolvendo tal trabalho, essas metas continuam sendo satisfeitas. Dessa forma, a avaliação de demandas de trabalho como estressores sofreria pouca influência de valores do trabalho, os quais teriam um impacto muito mais importante na escolha dos caminhos profissionais a serem traçados.
De modo geral, os estudos sobre os valores relativos ao trabalho e sobre sua relação com variáveis organizacionais começaram a crescer a partir da última década. Só recentemente, Ros e cols. (1999) desenvolveram uma teoria de valores relativos ao trabalho compatível com a teoria dos valores básicos individuais de Schwartz e, posteriormente, Porto e Tamayo (2003) adaptaram o modelo para o Brasil, desenvolvendo e validando um instrumento de medida para o construto. Assim, foram encontrados poucos estudos sobre a relação entre comportamentos e percepções no trabalho e os valores laborais e, principalmente, estudos que tivessem utilizado uma teoria de valores do trabalho integrada a uma teoria de valores individuais básicos. Quanto à relação entre valores do trabalho e estresse ocupacional, não foram encontradas pesquisas anteriores, o que impede a comparação de resultados. A pesquisa de Tamayo (2001) que encontrou relação entre valores e estresse ocupacional abordou os valores pessoais básicos e não os valores do trabalho.

Novas pesquisas sobre valores do trabalho e sobre a relação desta variável com estresse ocupacional são necessárias para que haja comparação de resultados e elementos que possam fundamentar ou refutar as hipóteses apresentadas para os resultados encontrados no presente estudo. Pesquisas futuras poderiam também verificar se outros tipos de interação entre trabalho e família, como impactos positivos da família sobre o trabalho, estariam relacionados com o estresse ocupacional.

\section{Referências}

Arciniega, L. M. \& González, L. (2002). What is the influence of work values relative to other variables in the development of organizational commitment?. Em A. Sagie \& M. Stasiak (Orgs.), Work values and behaviour in an era of transformation, $8^{\text {th }} \mathrm{Bi}$ annual Conference of the International Society for the Study of Work and Organizational Values (pp. 13-20). Poland: Academy of Humanities and Economics.

Beehr, T. A. (1998). Research on occupational stress: An unfinished enterprise. Personnel Psychology, 51(4), 835-844.

Browner, C. H. (1987). Job stress and health: The role of social support at work. Research in Nursing and Health, 10(1), 93-100.

Caplan, R. D. \& Jones, K. W. (1975). Effects of workload, role ambiguity, and Type A personality on anxiety, depression, and heart rate. Journal of Applied Psychology, 60(4), 713-719.

CARNET: The Canadian Aging Research Network (1993). Work and family: The survey findings for the work and eldercare research group. Guelph, ON: author.

Cooper, C. L., Dewe, Ph. J. \& O’Driscoll, M. P. (2001). Organizational stress: A review and critique of theory, research and applications. London: Sage Publications.

Cooper, C., Sloan, S. \& Williams, S. (1988). Occupational stress indicator management guide. London: Thorbay Press.

Edwards, J. R. (1992). A cybernetic theory of stress, coping, and well-being in organizations. The Academy of Management Review, 17(2), 238-274.

Edwards, J. R. \& Cooper, C. L. (1990). The person-environment fit approach to stress: Recurring problems and some suggested solutions. Journal of Organizational Behavior, 11(4), 293-307.

Edwards, J. R. \& Rothbard, N. P. (2000). Mechanisms linking work and family: Clarifying the relationship between work 
and family constructs. The Academy of Management Review, 25, 178-199.

Frone, M. R., Yardley, J. K. \& Markel, K. S. (1997). Developing and testing an integrative model of the work-family interface. Journal of Vocational Behavior, 50(2), 145-167.

Frone, M. R., Russell, M. \& Cooper, M. L. (1992). Antecedents and outcomes of work-family conflict: Testing a model of the work-family interface. Journal of Applied Psychology, 77(1), 65-78.

Iwata, N. \& Suzuki, K. (1997). Role stress - mental health relations in Japanese bank workers: A moderating effect of social support. Applied Psychology: An International Review, 45(2), 207-218.

Jamal, M. (1990). Relationship of job stress and type A behavior to employee's job satisfaction, organizational commitment, psychosomatic problems and turnover motivation. Human Relations, 43(8), 727-738.

Jex, S. M. (1998). Stress and job performance. London: Sage Publications.

Jex, S. M. \& Elacqua,T. C. (1999). Self-esteem as a moderator: A comparison of global and organization-based measures. Journal of Occupational and Organizational Psychology, 72(1), 71-81.

Jones, F. \& Kinman, G. (2001). Approaches to studying stress. Em F. Jones \& J. Bright (Orgs.), Stress: Myth, theory and research (pp. 17-45). England: Prentice Hall.

Kahn, R. L. \& Byosiere, P. (1992). Stress in organizations. Em M. D. Dunnete \& L. M. Hough (Orgs.), Handbook of industrial and organizational psychology (pp. 571-650). Palo Alto, CA: Consulting Psychologists Press.

Kelloway, E. K., Gottlieb, B. H. \& Barham, L. (1999). The source, nature, and direction of work and family conflict: A longitudinal investigation. Journal of Occupational Health Psychology, 4(4), 337-346.

Kirmeyer, A. \& Diamond, A. (1985). Coping by police officers: A study of role stress and type A and type B behavior patterns. Journal of Occupational Psychology, 52(3), 277-285.

Konrad, A. M. (2002). The relationship of work values to employment, hours of paid work, and family responsibilities: An analysis comparing women and men. Em A. Sagie \& M. Stasiak (Orgs.), Work values and behaviour in an era of transformation, 8th Bi-annual Conference of the International Society for the Study of Work and Organizational Values (pp. 234-240). Poland: Academy of Humanities and Economics.

Kunda, Z. (1999). Social cognition. Making sense of people. Cambridge: MIT Press.

Lazarus, R. S. (1995). Psychological stress in the workplace. Em R. Crandall, \& P. L. Perrewé (Orgs.), Occupational stress: A handbook (pp. 3-14). Washington, USA: Taylor \& Francis.
MacEwen, K. E. \& Barling, J. (1994). Maternal employment experiences affect children's behavior via mood, cognitive difficulties, and parenting. Journal of Marriage and the Family, 56(2), 507-510.

Marino, K. E. \& White, S. E. (1985). Departmental structure, locus of control, and job stress: The effect of moderators. Journal of Applied Psychology, 70(4), 782-784.

Paschoal, T. \& Tamayo, A. (2004). Validação da Escala de Estresse no Trabalho. Estudos de Psicologia, 9(1), 45-52.

Paschoal, T., Tamayo, A. \& Barham, E. J. (2002). Escala de Interação Trabalho-Família [Resumo]. Em Sociedade Brasileira de Psicologia (Org.), Resumos de comunicações científicas, XXXII Reunião Anual de Psicologia (pp. 318-319). Florianópolis: SBP.

Perry-Jenkins, M., Repetti, R. L. \& Crouter, A. C. (2000). Work and family in the 1990s. Journal of Marriage \& the Family, 62(4), 981-999.

Polasky, L. J. \& Holahan, C. K. (1998). Maternal self-discrepancies, inter-role conflict, and negative affect among married professional women with children. Journal of Family Psychology, 12(3), 388-401.

Porto, J. B. \& Tamayo, A. (2003). Escala de valores relativos ao trabalho - EVT. Psicologia: Teoria e Pesquisa, 19(2), 45-152.

Ros, M., Schwartz, S. H. \& Surkiss. S. (1999). Basic individual values, work values, and the meaning of work. Applied Psychology: An International Review, 48(1), 49-71.

Schmied, L. A. \& Lawler, K. A. (1986). Hardiness, Type A behavior, and the stress illness relation in working woman. Journal of Personality and Social Psychology, 51(6), 1218-1223.

Tamayo, A. (2001). Prioridades axiológicas, atividade física e estresse ocupacional. Revista de Administração Contemporânea, 5(3), 127-147.

Tamayo, A., Lima, D. \& da Silva, A. V. (2002). Impacto do clima organizacional sobre o estresse no trabalho. Trabalho apresentado no XXVI Encontro Nacional da ANPAD, Salvador, Bahia.

Thompson, L. \& Walker, A. (1989). Gender in families: Women and men in marriage, work and parenthood. Journal of Marriage and the Family, 45(2), 240-251.

Recebido em 07.06.2004

Primeira decisão editorial em 25.03.2005

Versão final em 29.04.2005

Aceito em 08.07.2005 